\title{
Long noncoding RNA MACC1-AS1 is a potential sponge of microRNA-34a in cervical squamous cell carcinoma and upregulates cyclin-dependent kinase 6
}

\author{
JIEQIONG JIN ${ }^{1 *}$, XUEMEI CHEN ${ }^{2 *}$, JING CHEN $^{3}$ and XIN GENG ${ }^{4}$ \\ ${ }^{1}$ Department of Gynaecology, Qingdao Jiaozhou Central Hospital, Qingdao, Shandong 266300; \\ ${ }^{2}$ Department of Obstetrics and Gynecology, Wuhan Third Hospital (Tongren Hospital of Wuhan University), Wuhan, \\ Hubei 430060; ${ }^{3}$ Department of Gynecological Oncology, Qingdao Center Medical Group, Qingdao, Shandong 266042; \\ ${ }^{4}$ Department of Gynaecology, Qingdao No. 6 People's Hospital, Qingdao, Shandong 266033, P.R. China
}

Received June 11, 2019; Accepted November 15, 2019

DOI: $10.3892 / \mathrm{ol} .2020 .11346$

\begin{abstract}
The present study aimed to investigate the role of long noncoding RNA MACC1-AS1 in cervical squamous cell carcinoma (CSCC). In the present study MACC1-AS1 expression as analyzed using reverse transcription-quantitative PCR. The interactions between MACC1-AS1 and miR-34a was analyzed via overexpression experiments. Cell cycle and proliferation analyses were performed to analyze the roles of MACC1-AS1 in regulating cancer cell cycle progression and cell proliferation. It was observed that MACC1-AS1 was upregulated in CSCC, and its expression levels were elevated with the increase in clinical stage. Bioinformatics analysis revealed that MACC1-AS1 may be a sponge of miR-34a, which can target cyclin-dependent kinase 6 (CDK6). In CSCC cells, MACC1-AS1 overexpression led to upregulation of CDK6, while miR-34a overexpression had the opposite effect and reduced the effects of MACC1-AS1 overexpression in co-transfected cells. Cell cycle and proliferation analyses demonstrated that MACC1-AS1 and CDK6 promoted cell cycle progression and cell proliferation. By contrast, miR-34a had the opposite effect on cell cycle proliferation and cell proliferation, reducing the effects induced by MACC1-AS1 overexpression. Therefore, the IncRNA MACC1-AS1 may serve as a sponge of miR-34a to upregulate CDK6, thereby promoting cell cycle progression and cell proliferation.
\end{abstract}

Correspondence to: Dr Xin Geng, Department of Gynaecology, Qingdao No. 6 People's Hospital, 9 Fushun Road, Qingdao, Shandong 266033, P.R. China

E-mail: yrcocyrlrp81@163.com

*Contributed equally

Key words: MACC1-AS1, cervical squamous cell carcinoma, microRNA-34a, cyclin-dependent kinase 6

\section{Introduction}

In 2018, a total of 570,000 new cases of cervical cancer and 311,000 associated mortalities were reported, and this malignancy is the fourth most common type of cancer in terms of incidence and mortality rates (1). Cervical squamous cell carcinoma (CSCC) is the most common histopathological form of cervical cancer and accounts for $~ 90 \%$ of all reported cases $(2,3)$. Human papillomavirus (HPV) infection is the leading cause of CSCC (4). With the popularization of HPV screening and vaccination program, the incidence of HPV-positive CSCC has dropped significantly during the past years (5). However, HPV vaccines cannot improve the conditions of patients who have already been infected (5). In addition, HPV-negative CSCC is more aggressive, and effective prevention and treatment approaches are currently lacking (6).

Genetic studies have revealed a considerable number of genetic factors with critical roles in CSCC (7).Cyclin-dependent kinase 6 (CDK6), a member of the CDK family, mainly regulates cell cycle progression in G1 phase (8). In CSCC, CDK6 is overexpressed and accelerates cell cycle progression of cancer cells to promote cancer progression (9). Therefore, inactivation of CDK6 is a promising strategy for the treatment of different types of cancer (10).

Specific tumor-suppressive microRNAs (miRNAs), such as $\mathrm{miR}-34 \mathrm{a}$, have been demonstrated to target and cleave CDK6, thereby inhibiting tumor growth (11). MACC1-AS1 is a long noncoding RNA (lncRNA), an RNA with a length of $>200$ nucleotides, that has been reported to have oncogenic functions only in gastric cancer $(12,13)$. In the present study, bioinformatics analysis revealed that MACC1-AS1 may form a base pair with miR-34a. The study aimed to investigate the interactions among MACC1-AS1, miR-34a and CDK6 in CSCC.

\section{Materials and methods}

CSCC patients. In the present study, a total of $60 \mathrm{CSCC}$ patients [including 39 males and 21 females; age range, 
40-66 years; mean age, 51.9 $\pm 6.6(\mathrm{SD})$ years] were selected from 111 CSCC cases diagnosed in Qingdao No. 6 People's Hospital (Qingdao) between March 2015 and April 2018. The present study was approved by the review board of the Ethics Committee of Qingdao No. 6 People's Hospital. Patients were included into the present study if they were newly diagnosed CSCC cases, had not received any prior cancer therapies, and no other therapies were initiated with 100 days prior to the admission day. The exclusion criteria included diagnosis of multiple clinical disorders, recurrent cases and history of other malignancies. Based on the clinical findings and American Joint Committee on Cancer (AJCC) staging system (14), a total of 12, 12, 16 and 20 of the included cases were classified as clinical stage I, II, III, and IV, respectively. Among the 60 CSCC patients, 46 cases were HPV-positive (including infection with HPV types 11, 16, and 18). All patients were informed of the contents of the present study and the potential publication of this paper, and all participants signed an informed consent form.

CSCC tissues and cells. Prior to the initiation of any therapies, a cervical biopsy was performed under the guidance of magnetic resonance imaging. During the biopsy, CSCC and adjacent non-tumor (within $2 \mathrm{~cm}$ around the tumors) tissues were obtained from the patients. The weight of each sample ranged between 0.014 and $0.019 \mathrm{~g}$. All tissue samples were subjected to histopathological tests to confirm that they were tumor or non-tumor samples. All tissue samples were stored at $-80^{\circ} \mathrm{C}$ before RNA extractions.

In addition, the SiHa human CSCC cell line (ATCC) was used in the present study. SiHa cells were cultured in a mixture of $10 \%$ fetal bovine serum and $90 \%$ Eagle's minimum essential medium under the conditions of $5 \% \mathrm{CO}_{2}, 95 \%$ humidity and $37^{\circ} \mathrm{C}$.

Cell transfection. miR-34a mimic (5'-UGGCAGUGUCUU AGCUGGUUGU-3') and negative control (NC) mimic (5'-CGCGAUUGUAAACUUGCCGCG-3') were obtained from GenePharma Co., Ltd. MACC1-AS1 and CDK6 expression vectors were established using the pcDNA3.1 vector (GenePharma Co., Ltd.). In order to perform transient transfections, SiHa cells were harvested when $80 \%$ confluence was reached and counted. Next, $2 \times 10^{6}$ cells in $2 \mathrm{ml}$ medium (10\% fetal bovine serum and $90 \%$ Eagle's minimum essential medium) were transferred to each well of a 6-well plate and were incubated with the transfection mixture, containing Lipofectamine $^{\circledR} 2000$ (Thermo Fisher Scientific, Inc.) and $40 \mathrm{nM} \mathrm{miR}-34 \mathrm{a}$ mimic or $10 \mathrm{nM}$ overexpression vector, for $5 \mathrm{~h}$ at $37^{\circ} \mathrm{C}$. NC mimic or empty vector served as the NC groups. Following transfection, the cells were washed with fresh cell culture medium. Untransfected cells served as the normal control cells in all transfection experiments. All subsequent experiments were performed using cells collected at $24 \mathrm{~h}$ post-transfection.

RNA extraction. Total RNAs were extracted from $2 \times 10^{5}$ cells (harvested at $24 \mathrm{~h}$ post-transfection) or $0.015 \mathrm{~g}$ tissue samples (ground in liquid nitrogen) using RiboZol (Sigma-Aldrich; Merck KGaA). To retain the miRNAs in RNA samples, $85 \%$ of ethanol was used to precipitate and wash the RNAs. All
RNA samples were subjected to digestion with DNase I for $2 \mathrm{~h}$ at $37^{\circ} \mathrm{C}$ to remove genomic DNA.

Reverse transcription-quantitative polymerase chain reaction $(q P C R)$. For mRNA detection, the digested RNA samples were reverse transcribed into cDNAs using Tetro Reverse Transcriptase (Bioline), and TB Green Advantage qPCR Premix (Clontech; Takara Bio USA, Inc.) was used in the qPCR mixture. The mRNA expression levels of MACC1-AS1 and CDK6 were measured by qPCR, with GAPDH serving as the endogenous control. For miRNA detection, the All-in-One ${ }^{\mathrm{TM}}$ miRNA qRT-PCR Detection kit (GeneCopoeia, Inc.) was used to perform addition of poly (A), reverse transcription and qPCR assays. The expression levels of miR-34a were measured, with U6 serving as the endogenous control. The thermal cycling conditions for all reactions were as follows: $95^{\circ} \mathrm{C}$ for $30 \mathrm{~min}$, followed by 40 cycles of $95^{\circ} \mathrm{C}$ for $10 \mathrm{sec}$ and $55^{\circ} \mathrm{C}$ for $40 \mathrm{sec}$. The following primer sequences were used in qPCR assays: MACC1-AS1 forward 5'-GCCAGTCAGAAA ATGAGGAAC-3' and reverse, 5'-CCAGTTGGGTGAACA GGAC-3'; CDK6 forward 5'-TGGAGACCTTCGAGCACC-3' and reverse, 5'-CACTCCAGGCTCTGGAACTT-3'; GAPDH forward 5'-CATCACTGCCACCCAG-3' and reverse 5'-ATG CCAGTGAGCTTCCC-3'; miR-34a forward 5'-CCGGCA TGGCAGTGTCTTAGCT-3' and reverse, 5'-CCAGTGCAG GGTCCGAGGTA-3'; and U6 forward 5'-CTCGCTTCGGCA GCACA-3' and reverse, 5'-AACGCTTCACGAATTGCGT-3'. The $2^{-\Delta \Delta \mathrm{Cq}}$ method (15) was used for gene expression normalization, and all qPCR experiments were performed in three replicates.

LncRNA-miRNA interaction prediction. The interacton between MACC1-AS1 and miR-34a was analyzed using IntaRNA 2.0 (http://rna.informatik.uni-freiburg. de/IntaRNA/Input.jsp) (16). In the analysis, the sequence of miR-34a was used as short sequence and sequence of MACC1-AS1 was used as long sequence. All other parameters were set as the default.

Western blot analysis. Total proteins were extracted from $2 \times 10^{5}$ cells (harvested at $24 \mathrm{~h}$ post-transfection) using RIPA solution and quantified using a BCA kit (both from GenePharma Co., Ltd.). For protein denaturation, all protein samples were incubated in boiling water for $8 \mathrm{~min}$. The denatured protein samples were then subjected to $10 \%$ SDS-PAGE, followed by gel transfer to PVDF membranes and blocking in 5\% non-fat milk in PBS for $90 \mathrm{~min}$ at room temperature. To detect the protein expression of CDK6, the membranes were incubated with rabbit anti-CDK6 $(1: 1,300$; ab226349; Abcam) and anti-GAPDH (serving as an endogenous control; 1:1,300; ab37168; Abcam) primary antibodies at $4^{\circ} \mathrm{C}$ for $18 \mathrm{~h}$. Next, the membranes were further incubated with horseradish peroxidase-conjugated IgG sercondary antibody (1:1,300; ab6721; Abcam), and this incubation was performed for $2 \mathrm{~h}$ at $24^{\circ} \mathrm{C}$. An enhanced chemiluminescence reagent (Sigma-Aldrich; Merck KGaA) was used to incubate the membranes for $10 \mathrm{~min}$ to develop the signals, and all data were quantified and normalized using ImageJ software, version 1.46 (National Institutes of Health, Bethesda, MD, USA). 
A

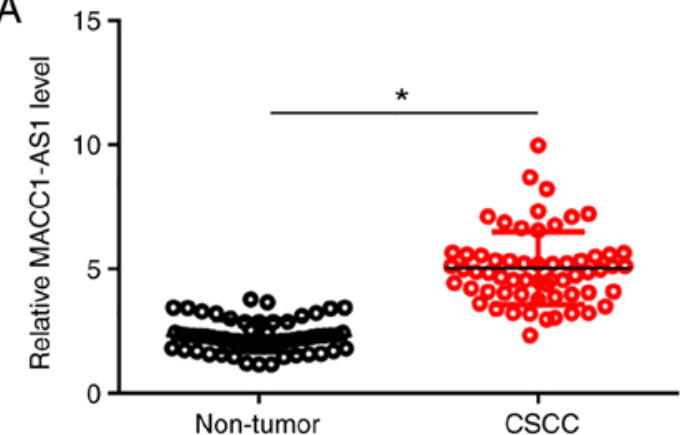

Non-tumor
B

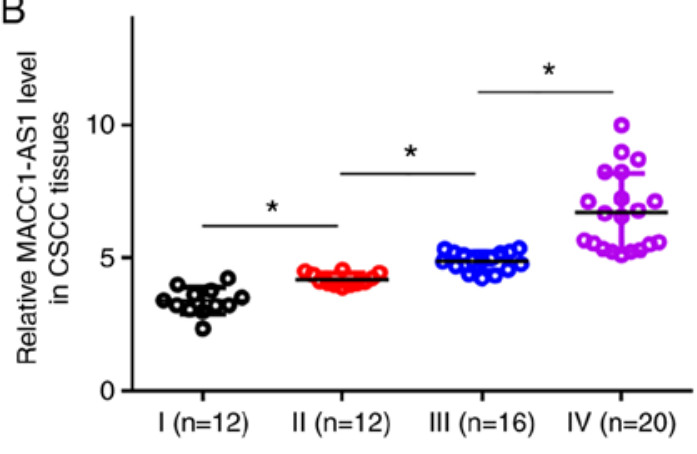

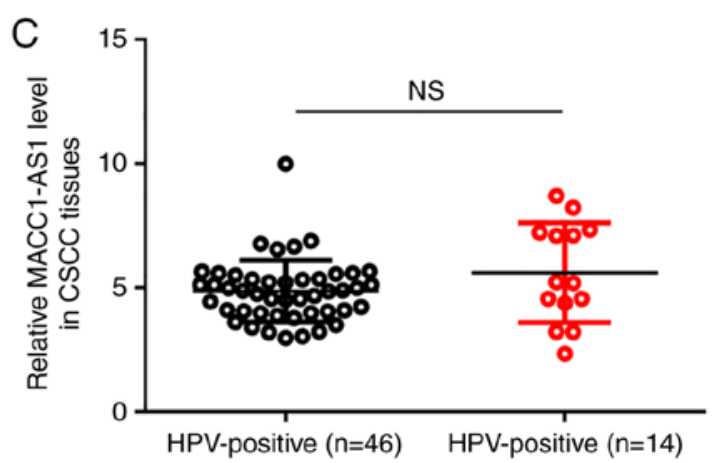

Figure 1. Upregulation of MACC1-AS1 was affected by the clinical stage, but not by HPV infection. (A) MACC1-AS1 expression in non-tumor and CSCC tissues was measured by reverse transcription-quantitative polymerase chain reaction and compared by paired t-test. (B) Expression levels of MACC1-AS1 in CSCC tissues were compared among four clinical stages by performing one-way analysis of variance in combination with Tukey's test. (C) Comparison of expression levels of MACC1-AS1 in CSCC tissues between HPV-negative and HPV-positive patients was performed by unpaired t-test. The mean values of three replicates are presented. ${ }^{*}<0.05$. HPV, human papillomavirus; CSCC, cervical squamous cell carcinoma; NS, not significant.

Cell Counting Kit-8 (CCK-8) cell proliferation analysis. Single-cell suspensions were prepared by mixing $4 \times 10^{3}$ cells with $1 \mathrm{ml}$ aforementioned culture medium (10\% fetal bovine serum and $90 \%$ Eagle's minimum essential medium). Cells were incubated in a 96-well culture plate with $0.1 \mathrm{ml}$ per well under the aforementioned cell culture conditions. Cells were collected every $24 \mathrm{~h}$ for a total of 4 days. At $4 \mathrm{~h}$ before the collection of cells, $10 \mu \mathrm{l}$ CCK-8 solution (Sigma-Aldrich; Merck KGaA) was added to each well. Finally, the optical density values at $450 \mathrm{nM}$ were measured.

Cell cycle analysis. SiHa cells were collected at $24 \mathrm{~h}$ post-transfection and were subjected to trypsinization. Cells were resuspended in pre-cold PBS, followed by centrifugation at $1,200 \mathrm{x} \mathrm{g}$ for $10 \mathrm{~min}$ at $4^{\circ} \mathrm{C}$. The supernatant was removed, and the cell pellets were resuspended in $75 \%$ ethanol. Following incubation in $75 \%$ ethanol for $4 \mathrm{~h}$ at $4^{\circ} \mathrm{C}$, the cells were centrifuged at $1,200 \mathrm{x} \mathrm{g}$ for $10 \mathrm{~min}$ at $4^{\circ} \mathrm{C}$. Next, the supernatant was removed, and the cell pellets were resuspended in pre-cold PBS. BD Pharmingen $^{\mathrm{TM}} \mathrm{PI} / \mathrm{RNa}$ e staining was then performed for $3 \mathrm{~min}$, and flow cytometer was conducted to separate the cells. The gating strategy was as follows: i) Define Gate 1: X-FSC; Y-SSC; Gate 2: X width; Y-FL2A; ii) cell debris and dead cells were excluded with ModFit LT (Verity Software House); and iii) a fluorescence analysis dot plot was then set up. 'Region 1-FSC vs. SSC', 'Zone 2 feasible' and 'Zone 3-Shared Mark' are on each corresponding fluorescent dot map. In each experiment, $10^{5}$ events were counted. Subsequently, Infinicyt ${ }^{\mathrm{TM}}$ flow cytometry data analysis software (ALPCO, Macedon, NY, USA) was used to analyze the data. A figure presenting the cell cycle data was plotted using Origin software, version 9.5 (OriginLab Corporation).

Statistical analysis. Experiments were performed in three replicates. Mean values were calculated and used in all data analyses. Assessment of differences between tissue types (non-tumor vs. CSCC) and among multiple cell transfection groups was performed by performing paired t-test or one-way analysis of variance (ANOVA) in combination with Tukey's test, respectively. Comparison between HPV-negative and HPV-positive CSCC patients was performed by unpaired t-test. $\mathrm{P}<0.05$ was considered to indicate a statistically significant difference.

\section{Results}

Upregulation of MACC1-AS1 is affected by clinical stage, but not by HPV infection status. The levels of MACC1-AS1 expression in the non-tumor and CSCC tissue samples were measured by RT-qPCR and compared by conducting paired t-test. It was observed that, compared with the non-tumor tissues, MACC1-AS1 expression was significantly higher in CSCC tissues (Fig. 1A; $\mathrm{P}<0.05$ ). Among the $60 \mathrm{CSCC}$ patients included in the present study, a total of 12, 12, 16 and 20 cases were classified as clinical stage I-IV, respectively. The expression levels of MACC1-AS1 were compared among the four stages by performing one-way ANOVA in combination with Tukey's test. With the increase of clinical stage, significantly increased expression levels of MACC1-AS1 were observed in CSCC tissues (Fig. 1B; P<0.05). Among the $60 \mathrm{CSCC}$ 
A
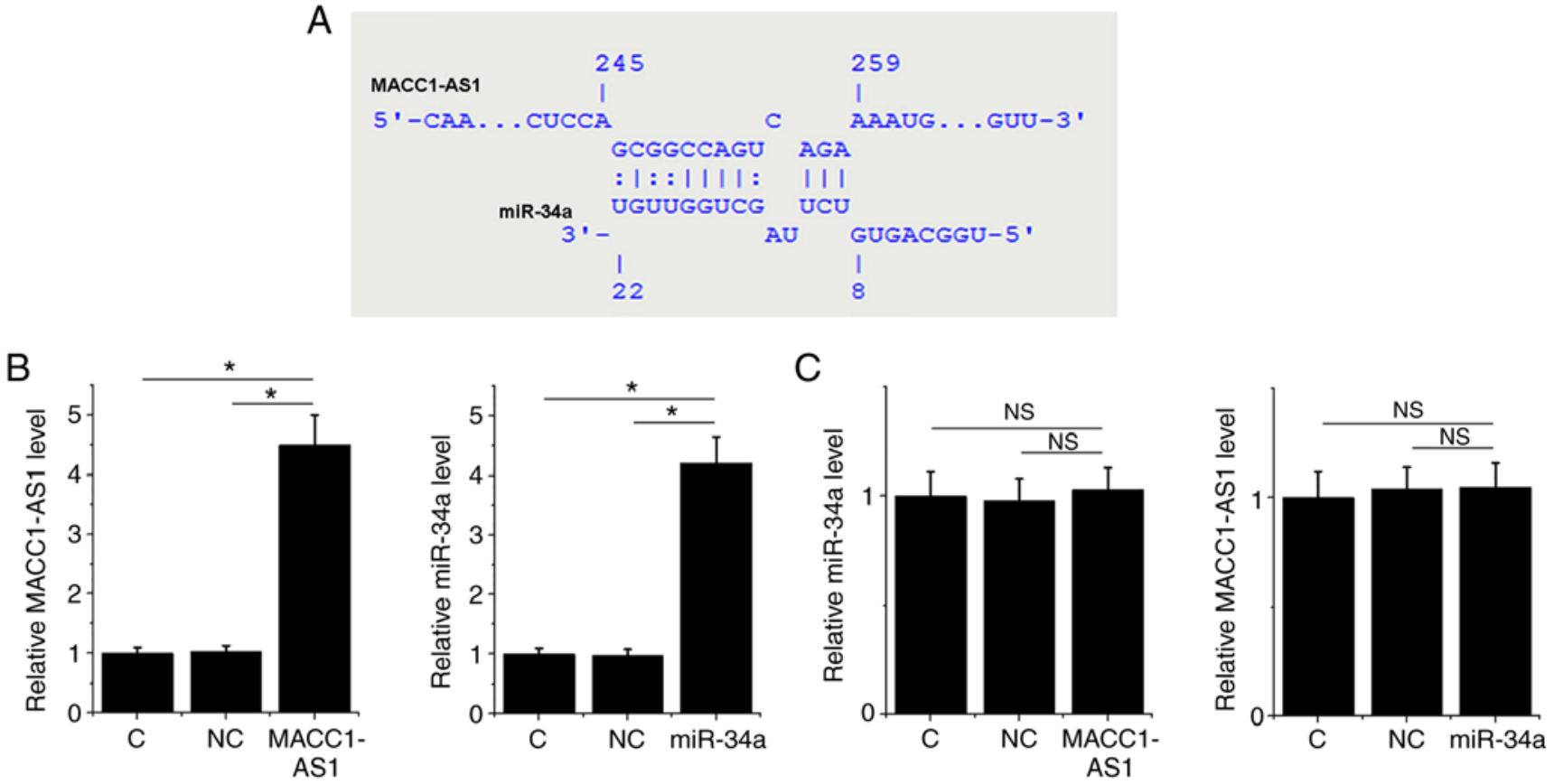

Figure 2. MACC1-AS1 may be a sponge of miR-34a. (A) Bioinformatics analysis using IntaRNA revealed that MACC1-AS1 forms base pairing with miR-34a Next, a MACC1-AS1 expression vector or miR-34a mimic was transfected into SiHa cells. (B) Overexpression of MACC1-AS1 and miR-34a in the corresponding transfected SiHa cells was confirmed by RT-qPCR at $24 \mathrm{~h}$ post-transfection. (C) The interaction between MACC1-AS1 and miR-34a was also analyzed by RT-qPCR. The mean values of three replicates are presented. " $\mathrm{P}<0.05$. miR, microRNA; RT-qPCR, reverse transcription-quantitative polymerase chain reaction; $\mathrm{C}$, normal control; $\mathrm{NC}$, negative control; $\mathrm{NS}$, not significant.

patients, 46 cases were HPV-positive (including infection with HPV types 11, 16 and 18). Comparison of expression levels of MACC1-AS1 in CSCC tissues between HPV-negative and HPV-positive patients was performed by unpaired t-test. The results revealed no significant difference between these two patient groups (Fig. 1C).

MACC1-AS1 may be a sponge of miR-34a. Bioinformatics analysis using the IntaRNA tool demonstrated that MACC1-AS1 forms base pairing with miR-34a (Fig. 2A). To further investigate the interaction between MACC1-AS1 and miR-34a, a MACC1-AS1 expression vector or miR-34a mimic was transfected into SiHa cells. At $24 \mathrm{~h}$ post-transfection, the RT-qPCR results revealed that, compared to the normal control and NC groups, the expression levels of MACC1-AS1 and miR-34a were significantly increased in their corresponding transfected $\mathrm{SiHa}$ cells, indicating successful overexpression (Fig. 2B; P<0.05). However, MACC1-AS1 overexpression did not affect the level of miR-34a, and similarly miR-34a overexpression did not significantly change the level of MACC1-AS1 (Fig. 2C; P>0.05).

MACC1-AS1 may sponge miR-34a to upregulate CDK6. It is known that CDK6 is a direct target of miR-34a (11). Western blot and RT-qPCR assays were performed to analyze the effects of the overexpression of miR-34a and CDK6 on CDK6 expression. As compared with the normal control and NC groups, MACC1-AS1 overexpression led to upregulated CDK6 at the mRNA (Fig. 3A) and protein (Fig. 3B) levels. By contrast, miR-34a overexpression had the opposite effect on CDK6 levels, while it reduced the effects of MACC1-AS1 overexpression in co-transfected cells (Fig. 3A and B).
MACC1-AS1 promoted SiHa cell proliferation and cell cycle progression via CDK6 and miR-34a. Cell proliferation and cell cycle assays were performed to analyze the effect of transfection on the proliferation and cell cycle progression of SiHa cells. Overexpression of CDK6 in vector-transfected cells was confirmed by western blot analysis (supplementary figure Fig. S1). Compared with the normal control and NC groups, overexpression of MACC1-AS1 or CDK6 significantly promoted cell proliferation (Fig. 4A; $\mathrm{P}<0.05$ ), while it also led to an increased cell percentage at $\mathrm{G} 2$ phase and decreased cell percentage at $\mathrm{G} 1$ phase (Fig. 4B and $\mathrm{C}$; $\mathrm{P}<0.05$ ). By contrast, miR-34a overexpression had the opposite effects on cell cycle progression and cell proliferation. In addition, miR-34a reduced the effects of MACC1-AS1 overexpression in cells co-transfected with MACC1-AS1 vector and miR-34a mimic (Fig. 4A-C). Therefore, MACC1-AS1 may upregulate CDK6 via miR-134a to promote $\mathrm{SiHa}$ cell proliferation and cell cycle progression.

\section{Discussion}

To date, the oncogenic function of MACC1-AS1 has only been analyzed in gastric cancer. It has been reported that MACC1-AS1 was upregulated in gastric cancer, and that overexpression of MACC1-AS1 was at least partially responsible for cancer cell metabolic plasticity and stemness $(12,13)$. However, to the best of our knowledge, the role of this lncRNA in other types of cancer remains unknown. The present study is, thus, the first to report the upregulation of MACC1-AS1 in CSCC tissues, and that MACC1-AS1 overexpression led to accelerated cell cycle progression and cell proliferation. In addition, the current study further revealed that MACC1-AS1 


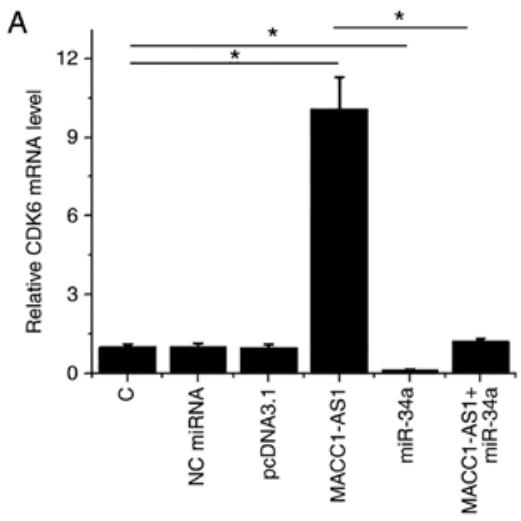

B
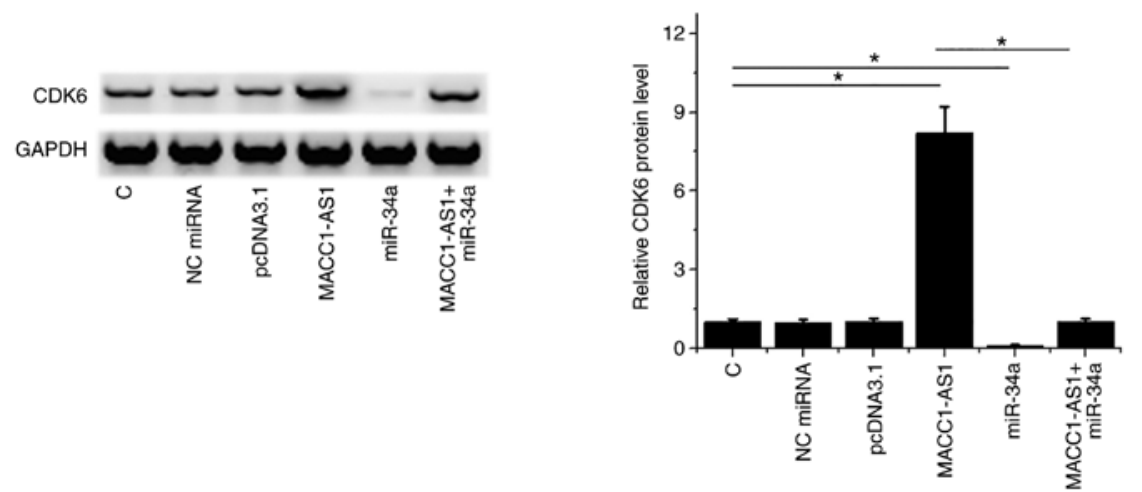

Figure 3. MACC1-AS1 may sponge miR-34a to upregulate CDK6. Reverse transcription-quantitative polymerase chain reaction and western blot analysis were performed to analyze the effects of the overexpression of miR-34a and/or MACC1-AS1 on CDK6 expression at the (A) mRNA and (B) protein levels, respectively. The mean values of three replicates are presented. ${ }^{*} \mathrm{P}<0.05$. miR, microRNA; CDK6, cyclin-dependent kinase 6; C, normal control; NC, negative control.
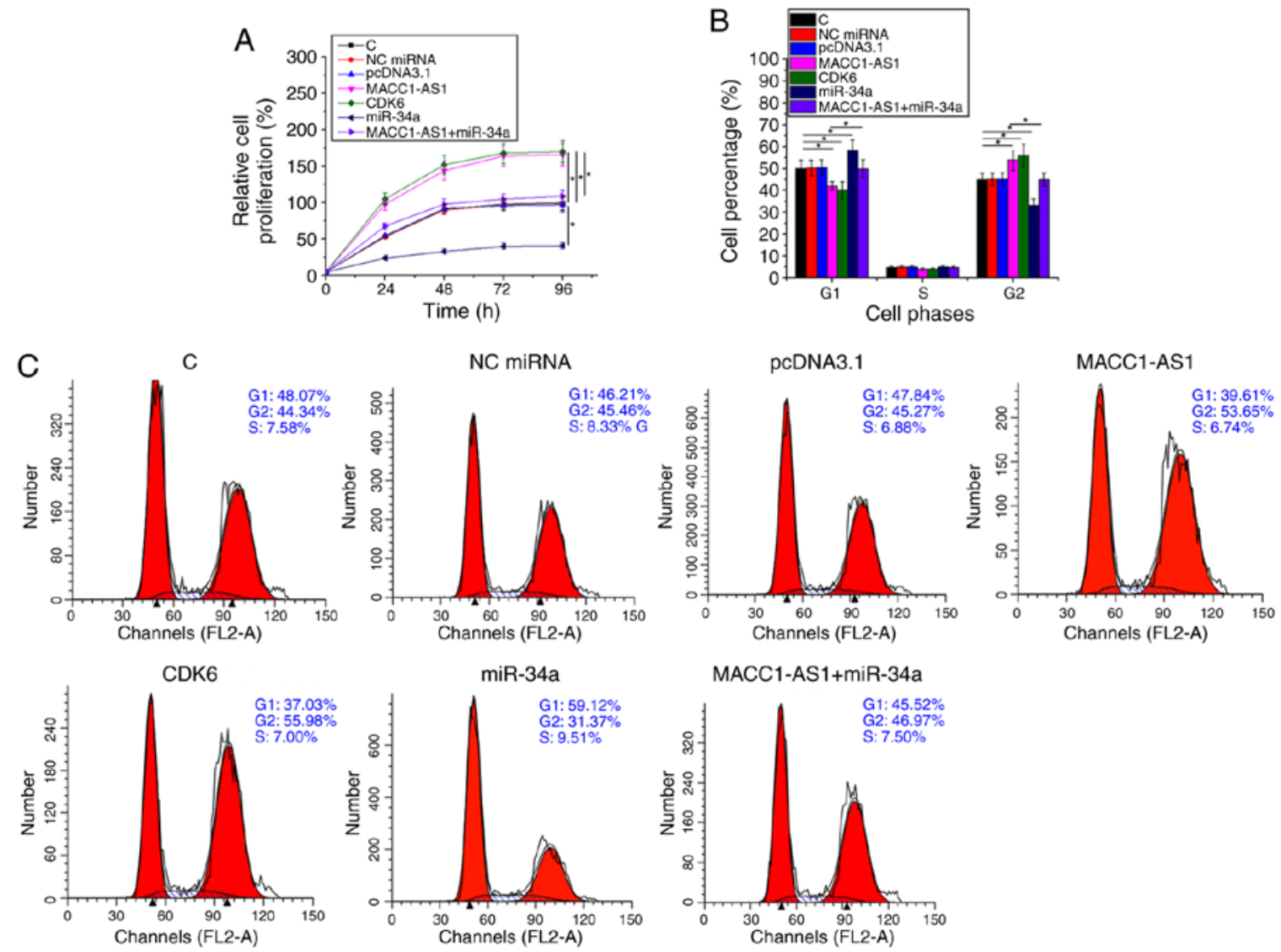

Figure 4. MACC1-AS1 promoted SiHa cell proliferation and cell cycle progression through regulation of CDK6 by sponging miR-34a. (A) Cell proliferation and (B) cell cycle assays were performed to analyze the effects of MACC1-AS1, miR-34a and CDK6 overexpression on the proliferation and cell cycle progression of SiHa cells. (C) Representative flow cytometry images are presented. "P<0.05. miR, microRNA; CDK6, cyclin-dependent kinase 6; C, normal control; NC, negative control. 
may serve as a sponge of miR-34a to upregulate CDK6, thereby promoting cell cycle progression and cell proliferation. Therefore, MACC1-AS1 may serve an oncogenic role in CSCC by promoting cell division.

The cancer stage is significantly associated with the survival of cancer patients (16). In the present study, increased expression levels of MACC1-AS1 were observed with the increase in CSCC clinical stage. This observation suggested that MACC1-AS1 expression may have a prognostic value in CSCC, and our future studies will perform follow-up analysis to evaluate this potential prognostic value of MACC1-AS1. Although HPV infection is the main cause of CSCC (17), the present study data suggested that MACC1-AS1 expression was not affected by HPV infection. Therefore, MACC1-AS1 may be involved in CSCC through HPV-independent pathways.

Previously, it has been reported that miR-34a can target CDK6 to suppress glioblastoma (11). In the present study, downregulation of CDK6 was observed in cells transfected with miR-34a mimic. Therefore, miR-34a may also target CDK6 in CSCC. Bioinformatics analysis further demonstrated that MACC1-AS1 forms base pairing with miR-34a. However, overexpression experiments revealed that MACC1-AS1 and miR-34a did not significantly affect the expression of each other, suggesting that miR-34a may not target MACC1-AS1. Previous studies on the interaction between IncRNAs and miRNAs indicated that lncRNAs may sponge miRNAs to upregulate their targets $(18,19)$. The data of the current study further revealed that MACC1-AS1 may sponge miR-34a to upregulate CDK6. It is worth noting that the bioinformatics analysis results indicated that MACC1-AS1 may sponge multiple miRNAs (data not shown), while most of these miRNAs are unlikely to be involved in CSCC.

According to the results, the present study revealed a novel MACC1-AS1/miR-34a/CDK6 axis in CSCC. The regulation of MACC1-AS1 expression may provide novel insights in CSCC treatment. It is worth noting that miR-34a in cancer biology may target multiple oncogenes, such as CD44 and MYCN $(20,21)$. Therefore, future studies will focus on the interactions between MACC1-AS1 and other targets of miR-34a. In addition, the bioinformatics analysis performed in the present study revealed that MACC1-AS1 interacted with multiple miRNAs, and these interactions should also be further investigated in future studies.

In conclusion, the current study revealed that MACC1-AS1 was upregulated in CSCC and that it may serve as a sponge of miR-34a to upregulate CDK6, thereby promoting the progression of CSCC.

\section{Acknowledgements}

Not applicable.

\section{Funding}

No funding was received.

\section{Availability of data and materials}

The datasets used and analyzed during the present study are available from the corresponding author on reasonable request.

\section{Authors' contributions}

JQJ and XMC performed the clinical studies, experimental work, data analysis and manuscript writing. JC performed the experimental work and literature research. XG was responsible for the study concept, research design and manuscript editing. All authors read and approved the final manuscript.

\section{Ethics approval and consent to participate}

Ethical approval was obtained from the Ethics Committee of Qingdao No. 6 People's Hospital (Qingdao, China). Written informed consent was signed by all participants.

\section{Patient consent for publication}

All patients provided written informed consent and the study was approved by the Ethics committee of the Qingdao No. 6 People's Hospital (Qingdao, China).

\section{Competing interests}

The authors declare that they have no competing interests.

\section{References}

1. Bray F, Ferlay J, Soerjomataram I, Siegel RL, Torre LA and Jemal A: Global cancer statistics 2018: GLOBOCAN estimates of incidence and mortality worldwide for 36 cancers in 185 countries. CA Cancer J Clin 68: 394-424, 2018.

2. Koh WJ, Greer BE, Abu-Rustum NR, Apte SM, Campos SM, Cho KR, Chu C, Cohn D, Crispens MA, Dorigo O, et al: Cervical cancer, version 2.2015. J Natl Compr Canc Netw 13: 395-404, 2015.

3. Small W Jr, Bacon MA, Bajaj A, Chuang LT, Fisher BJ, Harkenrider MM, Jhingran A, Kitchener HC, Mileshkin LR, Viswanathan AN and Gaffney DK: Cervical cancer: A global health crisis. Cancer 123: 2404-2412, 2017.

4. Wentzensen N, Schiffman M, Palmer T and Arbyn M: Triage of HPV positive women in cervical cancer screening. J Clin Virol 76 (Suppl 1): S49-S55, 2016.

5. Lowy DR: HPV vaccination to prevent cervical cancer and other HPV-associated disease: From basic science to effective interventions. J Clin Invest 126: 5-11, 2016.

6. Walboomers JM and Meijer CJ: Do HPV-negative cervical carcinomas exist? J Pathol 181: 253-254, 1997.

7. Cancer Genome Atlas Research Network; Albert Einstein College of Medicine; Analytical Biological Services; Barretos Cancer Hospital; Baylor College of Medicine; Beckman Research Institute of City of Hope; Buck Institute for Research on Aging; Canada's Michael Smith Genome Sciences Centre; Harvard Medical School; Helen F. Graham Cancer Center \&Research Institute at Christiana Care Health Services, et al: Integrated genomic and molecular characterization of cervical cancer. Nature 543: 378-384, 2017.

8. Swaffer MP, Jones AW, Flynn HR, Snijders AP and Nurse P: CDK substrate phosphorylation and ordering the cell cycle. Cell 167: 1750-1761.e16, 2016.

9. Shen SN, Wang H and Gong BL: Expressions of miRNA29 target genes CCND2 and CDK6 in cervical cancer. Disc Clin Cases 5: 14-18, 2018.

10. Sherr CJ, Beach D and Shapiro GI: Targeting CDK4 and CDK6: From discovery to therapy. Cancer Discov 6: 353-367, 2016.

11. Li Y, Guessous F, Zhang Y, Dipierro C, Kefas B, Johnson E, Marcinkiewicz L, Jiang J, Yang Y, Schmittgen TD, et al: MicroRNA-34a inhibits glioblastoma growth by targeting multiple oncogenes. Cancer Res 69: 7569-7576, 2009.

12. Zhao Y, Liu Y, Lin L, Huang Q, He W, Zhang S, Dong S, Wen Z, Rao J, Liao W and Shi M: The IncRNA MACC1-AS1 promotes gastric cancer cell metabolic plasticity via AMPK/Lin28 mediated mRNA stability of MACC1. Mol Cancer 17: 69, 2018. 
13. He W, Liang B, Wang C, Li S, Zhao Y, Huang Q, Liu Z, Yao Z, Wu Q, Liao W, et al: MSC-regulated lncRNA MACC1-AS1 promotes stemness and chemoresistance through fatty acid oxidation in gastric cancer. Oncogene 38: 4637-4654, 2019.

14. Livak KJ and Schmittgen TD: Analysis of relative gene expression data using real-time quantitative PCR and the 2(-Delta Delta C(T)) method. Methods 25: 402-408, 2001.

15. Busch A, Richter AS and Backofen R: IntaRNA: Efficient prediction of bacterial sRNA targets incorporating target site accessibility and seed regions. Bioinformatics 24: 2849-2856, 2008.

16. Edge SB and Compton CC: The American Joint Committee on Cancer: The 7th edition of the AJCC cancer staging manual and the future of TNM. Ann Surg Oncol 17: 1471-1474, 2010.

17. Strohl AE, Mendoza G, Ghant MS, Cameron KA, Simon MA, Schink JC and Marsh EE: Barriers to prevention: Knowledge of HPV, cervical cancer, and HPV vaccinations among African American women. Am J Obstet Gynecol 212: 65 e1-e5, 2015.

18. Paraskevopoulou MD and Hatzigeorgiou AG: Analyzing MiRNA-LncRNA Interactions. Methods Mol Biol 1402: 271-286, 2016.
19. Jalali S, Bhartiya D, Lalwani MK, Sivasubbu S and Scaria V: Systematic transcriptome wide analysis of 1ncRNA-miRNA interactions. PLoS One 8: e53823, 2013.

20. Liu C, Kelnar K, Liu B, Chen X, Calhoun-Davis T, Li H, Patrawala L, Yan H, Jeter C, Honorio S, et al: The microRNA miR-34a inhibits prostate cancer stem cells and metastasis by directly repressing CD44. Nat Med 17: 211-215, 2011.

21. Wei JS, Song YK, Durinck S, Chen QR, Cheuk AT, Tsang P Zhang Q, Thiele CJ, Slack A, Shohet J and Khan J: The MYCN oncogene is a direct target of miR-34a. Oncogene 27: 5204-5213, 2008.

(i) $(5)$ This work is licensed under a Creative Commons Attribution-NonCommercial-NoDerivatives 4.0 International (CC BY-NC-ND 4.0) License. 